\title{
STING-mediated inflammation in Kupffer cells contributes to progression of nonalcoholic steatohepatitis
}

\author{
Yongsheng Yu, ${ }^{1}$ Yu Liu, ${ }^{2}$ Weishuai An, ${ }^{1}$ Jingwen Song, ${ }^{1}$ Yuefan Zhang, ${ }^{3}$ and Xianxian Zhao ${ }^{1}$ \\ 'Department of Cardiovasology, Changhai Hospital, Second Military Medical University, Shanghai, China. ${ }^{2}$ Department of Cardiology, Nanjing University Medical School Affiliated Nanjing Drum Tower \\ Hospital, Nanjing, China. ${ }^{3}$ Department of Clinical Pharmacy, Shanghai General Hospital, Shanghai Jiaotong University, Shanghai, China.
}

\begin{abstract}
Innate immune activation contributes to the transition from nonalcoholic fatty liver to nonalcoholic steatohepatitis (NASH). Stimulator of IFN genes (STING, also referred to Tmem173) is a universal receptor that recognizes released DNA and triggers innate immune activation. In this work, we investigated the role of STINC in the progression of NASH in mice. Both methionine- and choline-deficient diet (MCD) and high-fat diet (HFD) were used to induce NASH in mice. Strikingly, STING deficiency attenuated steatosis, fibrosis, and inflammation in livers in both murine models of NASH. Additionally, STING deficiency increased fasting glucose levels in mice independently of insulin, but mitigated HFD-induced insulin resistance and weight gain and reduced levels of cholesterol, triglycerides, and LDL in serum; it also enhanced levels of HDL. The mitochondrial DNA (mtDNA) from hepatocytes of HFD-fed mice induced TNF- $\alpha$ and IL-6 expression in cultured Kupffer cells (KCs), which was attenuated by STING deficiency or pretreatment with BAY11-7082 (an NF-кB inhibitor). Finally, chronic exposure to 5,6-dimethylxanthenone-4-acetic acid (DMXAA, a STING agonist) led to hepatic steatosis and inflammation in WT mice, but not in STING-deficient mice. We proposed that STING functions as an mtDNA sensor in the KCs of liver under lipid overload and induces NF-кB-dependent inflammation in NASH.
\end{abstract}

\section{Introduction}

Nonalcoholic fatty liver disease (NAFLD) ranges from simple steatosis to nonalcoholic steatohepatitis (NASH), cirrhosis, and even hepatocellular carcinoma. Currently, the population prevalence of NAFLD in Asia is around 25\%, which is similar to that in many Western countries (1). The pathophysiology of NASH is still not fully understood, but it is commonly accepted in the field that innate immune activation is a key factor in initiating and amplifying hepatic inflammation, contributing to the transition from simple steatosis to NASH (2). The innate immune response was activated in leukocytes, such as Kupffer cells (KCs), by damage-associated molecular patterns (DAMPs) and pathogen-associated molecular patterns (PAMPs) $(2,3)$. Recently, the mitochondrial DNA (mtDNA) has been recognized as one of the endogenous DAMPs when released into the cytosol and extracellular environment, activating innate immune response and promoting inflammation $(4,5)$. NASH patients featured higher mitochondrial mass, but lower maximal respiration and increased oxidative DNA damage (6). Furthermore, it was reported that obese patients with high serum alanine aminotransferase (ALT) levels had increased plasma levels of mtDNA, but not nuclear DNA (nDNA) (7). Innate immune recognition relies on a pattern recognition receptor, such as TLR9, located in the endoplasmic reticulum. It was reported

Authorship note: YY and YL contributed equally to this work Conflict of interest: The authors have declared that no conflict of interest exists. License: Copyright 2019, American Society for Clinical Investigation.

Submitted: April 25, 2018; Accepted: November 6, 2018.

Reference information: / Clin Invest. 2019;129(2):546-555.

https://doi.org/10.1172/JCI121842. that hepatocyte-derived mtDNA activated TLR9 in the macrophages and induced expression of various inflammatory cytokines and chemokines, which, in turn, increased triglyceride accumulation in hepatocytes, activated fibrosis in the stellate cells, and thereby accelerated NASH progression $(7,8)$. In addition to interacting with the TLR9 pathway, mtDNA could also be detected by stimulator of IFN genes-dependent (STING-dependent, STING, also referred to as Tmem173, MPYS, MITA, and ERIS) cytosolic sensors $(9,10)$. Systemic injection of oxidized mtDNA enhanced IFN-stimulated gene expression in spleens of WT mice, but not STING-deficient mice (11). STING appeared to be a universal receptor for cyclic dinucleotides, including the bacterial second messengers 3'-5'-cyclic-di-adenosine-monophosphate (CDA) and 3'-5'-cyclic-di-guanosine-monophosphate (CDG) as well as the newly identified metazoan second messenger, cyclic-GMPAMP (12). STING deficiency prevented hepatic injury and fibrosis in both acute and chronic carbon tetrachloride-treated mice (13). Deficiency of STING prevented lipid accumulation in hepatocytes and protected from alcohol-induced liver injury at early time points (14). More importantly, it was reported that STING deficiency partially prevented high-fat diet-induced (HFDinduced) adipose tissue inflammation, obesity, insulin resistance, and glucose intolerance (15).

Thus, we hypothesized that deficiency of STING attenuates the progression of NASH and employed the mice that were fed with methionine- and choline-deficient diet (MCD) or HFD as murine models of NASH to investigate the role of STING. Mice fed a MCD diet rapidly (for about 8 weeks) develop steatohepatitis in livers, which mimics the impairment observed in patients with NASH, but it is associated with significant weight loss and 
A
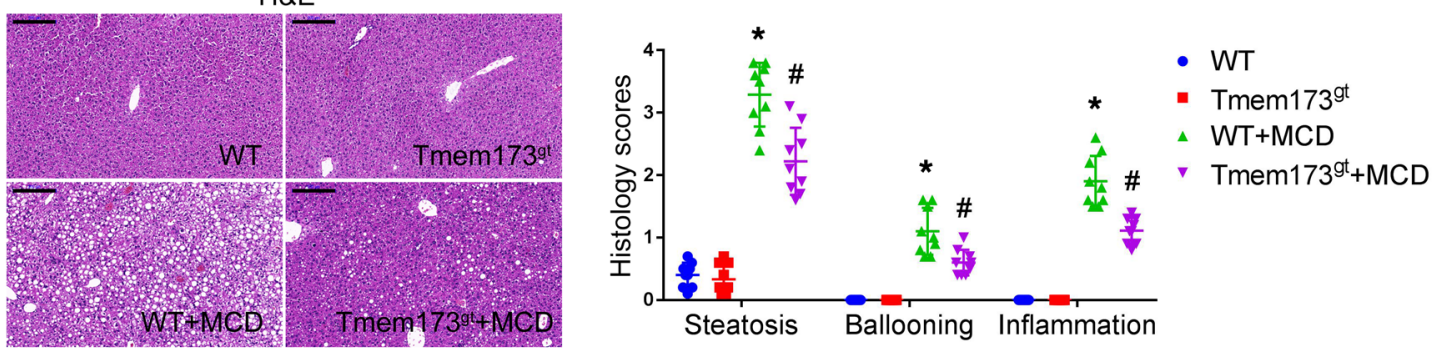

B
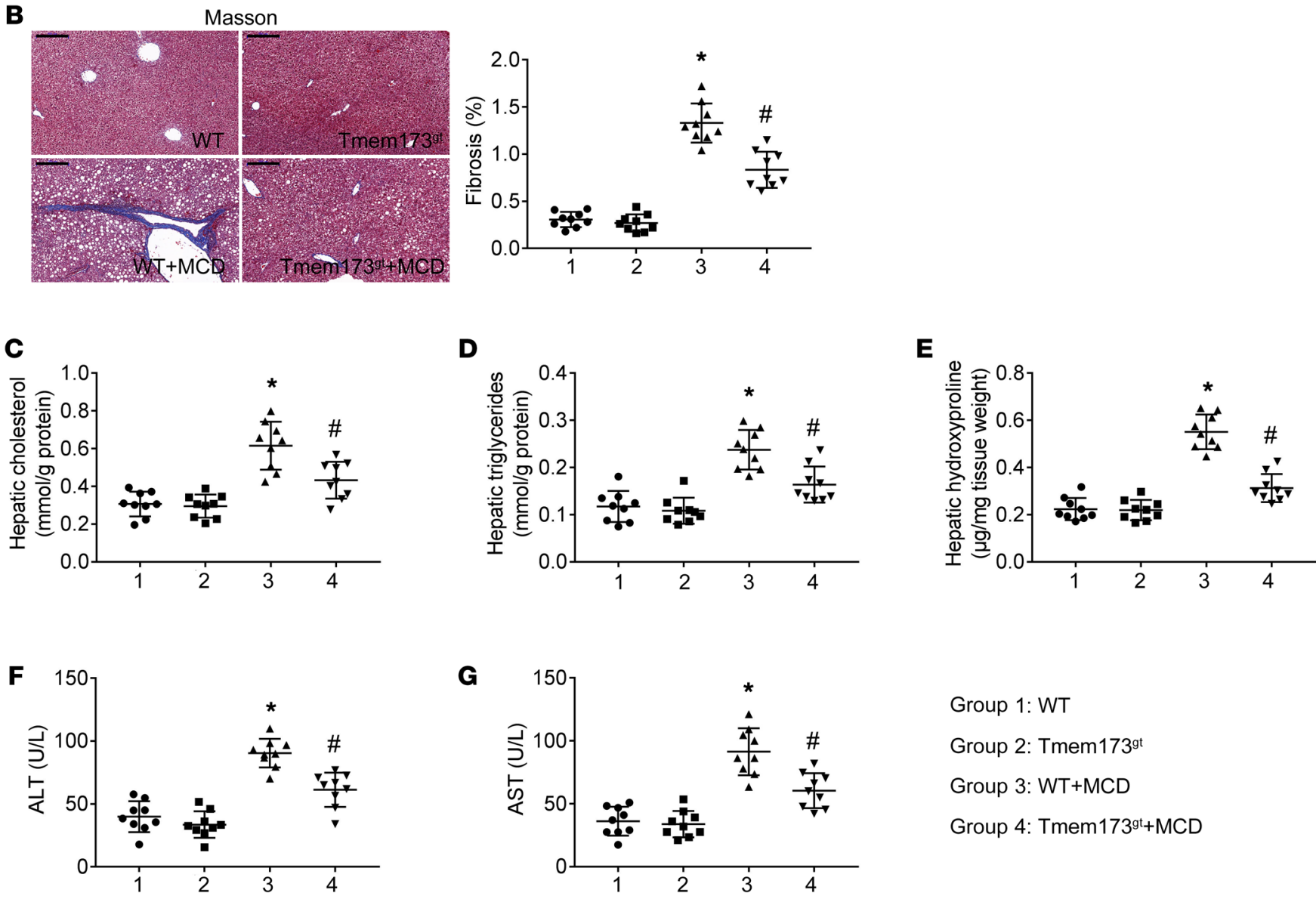

\author{
Group 1: WT \\ Group 2: Tmem173 ${ }^{\text {gt }}$ \\ Group 3: WT+MCD \\ Group 4: Tmem1739t+MCD
}

Figure 1. Deficiency of STING attenuated MCD-induced hepatic steatosis and fibrosis in mice. WT or STING-deficient mice (Tmem173"t) were exposed to MCD for 8 weeks to induce NASH. Paraffin-embedded liver sections were stained with H\&E (A) or Masson-trichrome (B), and hepatic histological scores and fibrosis area were calculated. Scale bars: $200 \mu \mathrm{m}$. Graphs showed levels of cholesterol (C), triglycerides (D), and hydroxyproline (E) in livers and levels of ALT (F) and AST (G) in serum. $n=9$ in each group. Values are shown as mean \pm SD. ${ }^{*} P<0.05$ versus WT mice fed with control diet; ${ }^{\#}<0.05$ versus WT mice fed with MCD. Statistical significance was determined using 1-way ANOVA followed by Tukey-Kramer multiple comparisons test.

the mice do not exhibit insulin resistance (16). NASH develops in HFD-fed mice and is linked to pathogenic factors similar to those in humans, with steatosis and metabolic syndrome preceding the transition to steatohepatitis, but it requires a long feeding period (17).

In addition, KCs were isolated from livers of WT or STINGdeficient (Tmem173"st) mice fed with chow or HFD. The mtDNA isolated from hepatocytes was added to these KCs to allow for investigation of the underlying mechanism by which STING deficiency protected against mtDNA-induced inflammation.

\section{Results}

Deficiency of STING attenuated MCD-induced hepatic steatosis and fibrosis in mice. WT and STING-deficient mice (Tmem173"st) were fed with MCD for 8 weeks to induce NASH. H\&E (Figure 1A) and Masson staining (Figure 1B) revealed steatosis, ballooning, inflammation, and fibrosis in the livers of MCD-fed mice, which was attenuated by deficiency of STING. Levels of cholesterol (Figure 1C), triglyceride (Figure 1D), and hydroxyproline (a marker of fibrosis, Figure 1E) in livers and levels of ALT (Figure 1F) and aspartate aminotransferase (AST) (Figure 1G) in serum were high- 
A

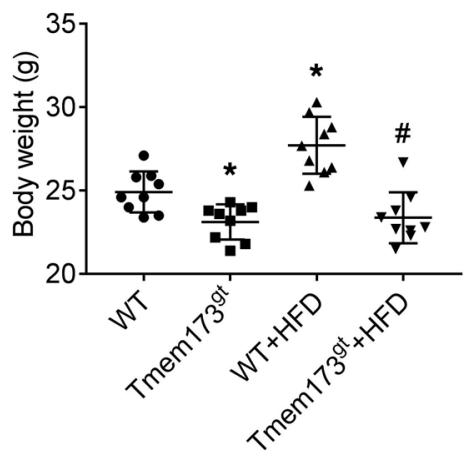

B

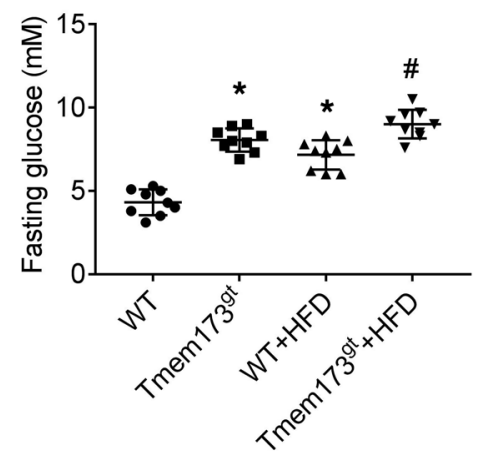

C

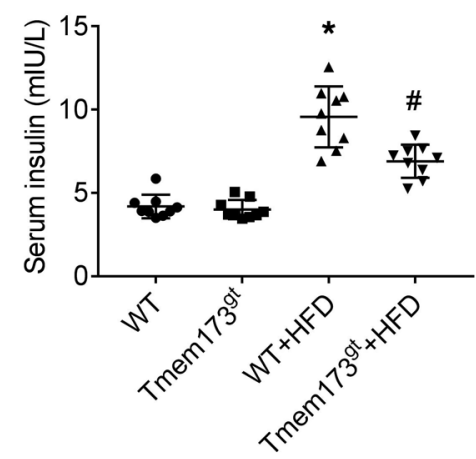

D
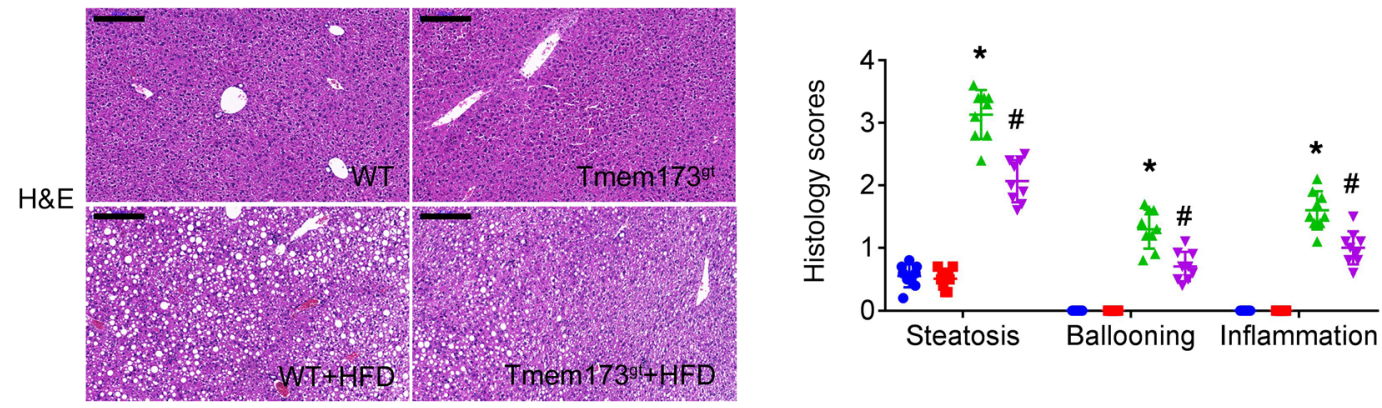

E

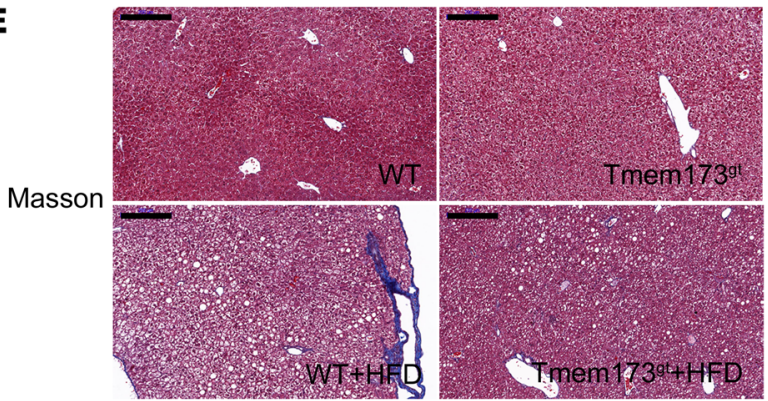

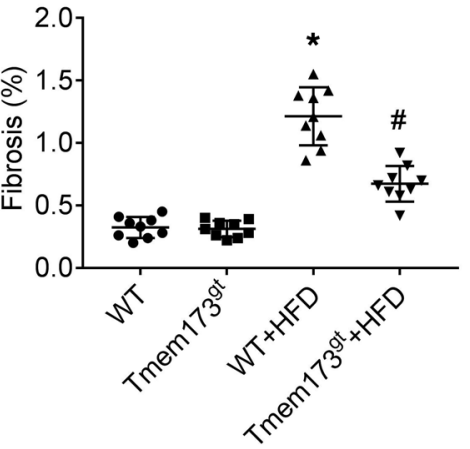

Figure 2. Deficiency of STING attenuated HFD-induced insulin resistance and histological change in livers of mice. WT or STING-deficient mice (Tmem173 ${ }^{\text {gt }}$ ) were exposed to HFD for 26 weeks to induce NASH. Graphs show the body weight (A) and levels of fasting glucose (B) and insulin (C) in serum. Paraffin-embedded liver sections were stained with H\&E (D) or Masson-trichrome (E), and hepatic histological scores and fibrosis area were calculated. Scale bars: $200 \mu \mathrm{m} . n$ = 9 in each group. Values are shown as mean \pm SD. ${ }^{*} P<0.05$ versus WT mice fed with control diet; ${ }^{\#}<0.05$ versus WT mice fed with HFD. Statistical significance was determined using 1-way ANOVA followed by Tukey-Kramer multiple comparisons test.

er in MCD-fed mice. STING deficiency lowered the levels of ALT and AST in serum and reduced levels of triglyceride, cholesterol, and hydroxyproline in livers when mice were fed with MCD.

In control mice, STING deficiency had no significant effect on levels of ALT or AST in serum and levels of triglyceride, cholesterol, or hydroxyproline in livers. In mice fed with control diet or MCD, STING deficiency had no significant effect on levels of cholesterol or triglyceride in serum (Supplemental Figure 1; supplemental material available online with this article; https://doi. org/10.1172/JCI121842DS1).

Deficiency of STING attenuated HFD-induced hepatic steatosis and fibrosis in mice. WT and STING-deficient mice were fed with HFD for 26 weeks to induce NASH. At the end of the experiment, body weight of STING-deficient mice was lower than that of WT mice (Figure 2A). Feeding with HFD resulted in a significant enhance- ment of body weight in WT mice, but not in STING-deficient mice. Fasting glucose levels of STING-deficient mice were higher than those in WT mice (Figure 2B). HFD led to a significant enhancement of fasting glucose levels of WT mice. HFD in STING-deficient mice slightly enhanced fasting glucose levels, but not significantly. The insulin levels were similar between WT and STING-deficient mice (Figure 2C). HFD resulted in a significant enhancement of insulin levels in WT mice, but not in STING-deficient mice, which indicated that deficiency of STING attenuated HFD-induced insulin resistance in mice. $\mathrm{H} \& \mathrm{E}$ (Figure 2D) and Masson staining (Figure $2 \mathrm{E})$ revealed steatosis, ballooning, inflammation, and fibrosis in the livers of HFD-fed mice, which was attenuated by STING deficiency.

Levels of cholesterol (Figure 3A), triglyceride (Figure 3B), and hydroxyproline (Figure 3C) in livers and levels of ALT (Figure 3D), AST (Figure 3E), cholesterol (Figure 3F), triglycerides (Figure 3G), 
A

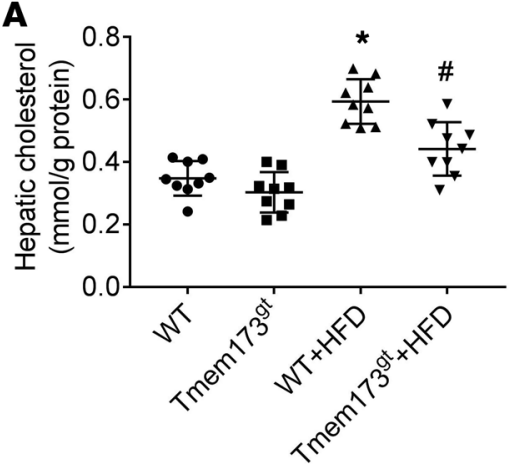

D

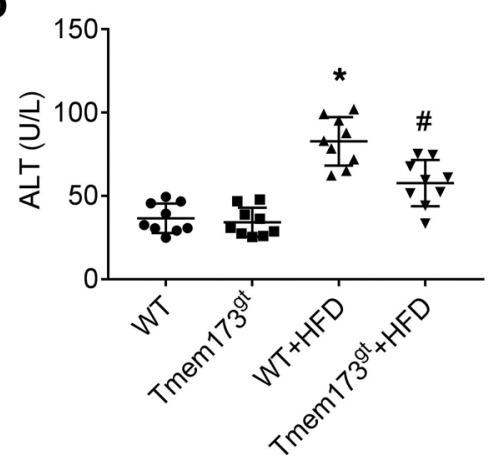

G

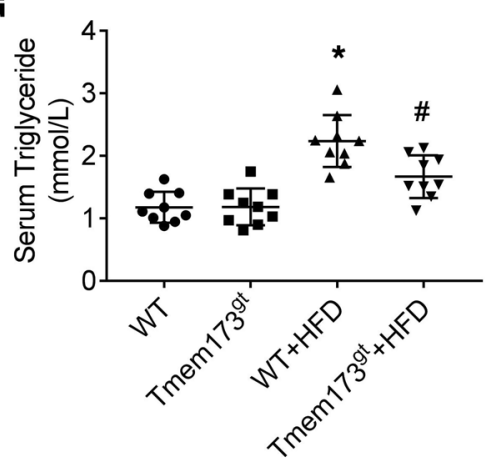

B

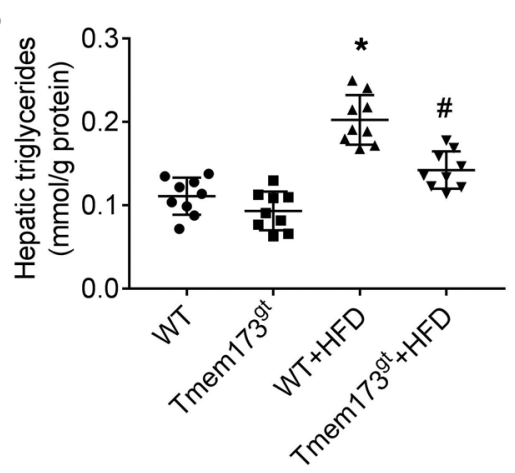

E

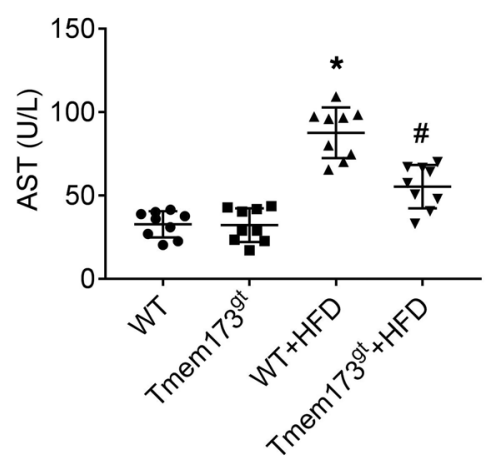

H

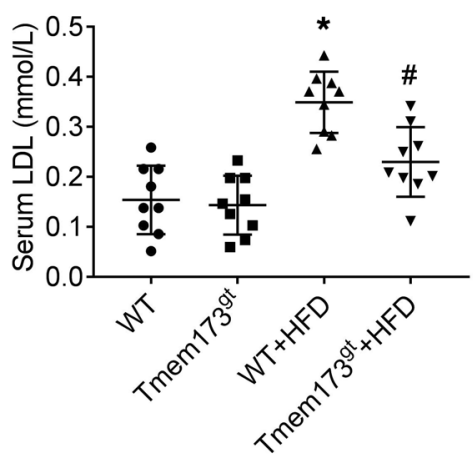

C

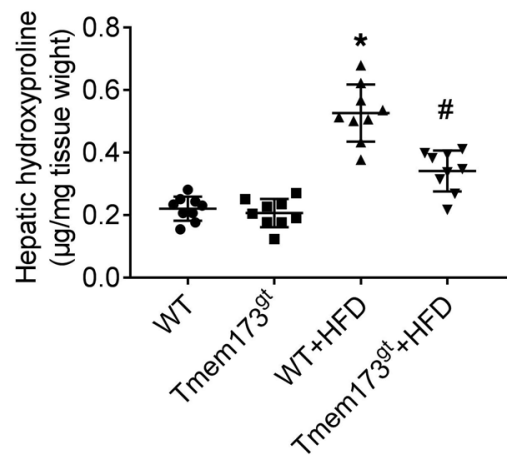

$\mathbf{F}$

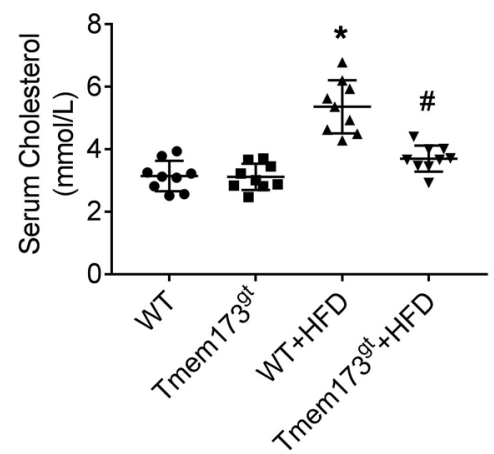

I

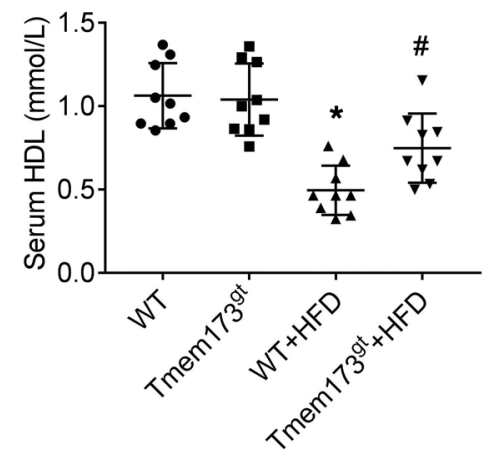

Figure 3. Deficiency of STING attenuated HFD-induced hepatic steatosis and fibrosis in mice. WT or STING-deficient mice (Tmem173 ${ }^{\text {gt }}$ ) were exposed to HFD for 26 weeks to induce NASH. Graphs show levels of cholesterol (A), triglycerides (B), and hydroxyproline (C) in livers and levels of ALT (D), AST (E), cholesterol $(\mathbf{F})$, triglycerides (G), LDL (H), and HDL (I) in serum. $n=9$ in each group. Values are shown as mean \pm SD. ${ }^{*} P<0.05$ versus WT mice fed with control diet; ${ }^{\#} P<$ 0.05 versus WT mice fed with HFD. Statistical significance was determined using 1-way ANOVA followed by Tukey-Kramer multiple comparisons test.

and LDL (Figure 3H) in sera were higher in HFD-fed mice. Levels of HDL (Figure 3I) in sera were lower in HFD-fed mice. STING deficiency in HFD-fed mice lowered the levels of ALT, AST, cholesterol, triglycerides, and LDL in sera, reduced levels of triglyceride, cholesterol, and hydroxyproline in livers, and enhanced levels of HDL in sera.

Deficiency of STING attenuated MCD- or HFD-induced inflammation in livers of mice. Both MCD (Figure 4A) and HFD (Figure 4B) led to upregulation of mRNA expression of F4/80, TNF- $\alpha$, and IL-6 in livers, which was alleviated by STING deficiency. Similar results were demonstrated in protein levels of TNF- $\alpha$ and IL-6 (Supplemental Figure 2). Both MCD and HFD led to upregulation of mRNA expression of IL-1 $\beta$ in livers. However, STING deficiency did not significantly affect IL-1 $\beta$ mRNA expression in livers.
To obtain further insight into steatosis and fibrosis, transcript levels of genes implicated in hepatic lipogenesis and fibrogenesis were evaluated. STING deficiency reduced mRNA levels of SREBP1c, FAS, and CD36 and enhanced mRNA levels of PPAR $\alpha$ in livers of mice fed with MCD or HFD (Supplemental Figure 3). In addition, STING deficiency significantly reduced mRNA levels of Col1A1 and $\alpha$-SMA in livers of mice fed with MCD or HFD (Figure 4, C and D).

We also tested the mRNA expression of type I IFN in livers of NASH models. In MCD-fed mice, IFN- $\alpha$ and IFN- $\beta$ mRNA levels were similar to those in control mice (Supplemental Figure $4 \mathrm{~A}$ ). In HFD-fed mice, IFN- $\alpha$ and IFN- $\beta$ mRNA levels were higher than those in control mice (Supplemental Figure 4B). STING deficiency had no significant effect on mRNA expression of IFN- $\alpha$ and IFN- $\beta$ in control mice or NASH mice. 

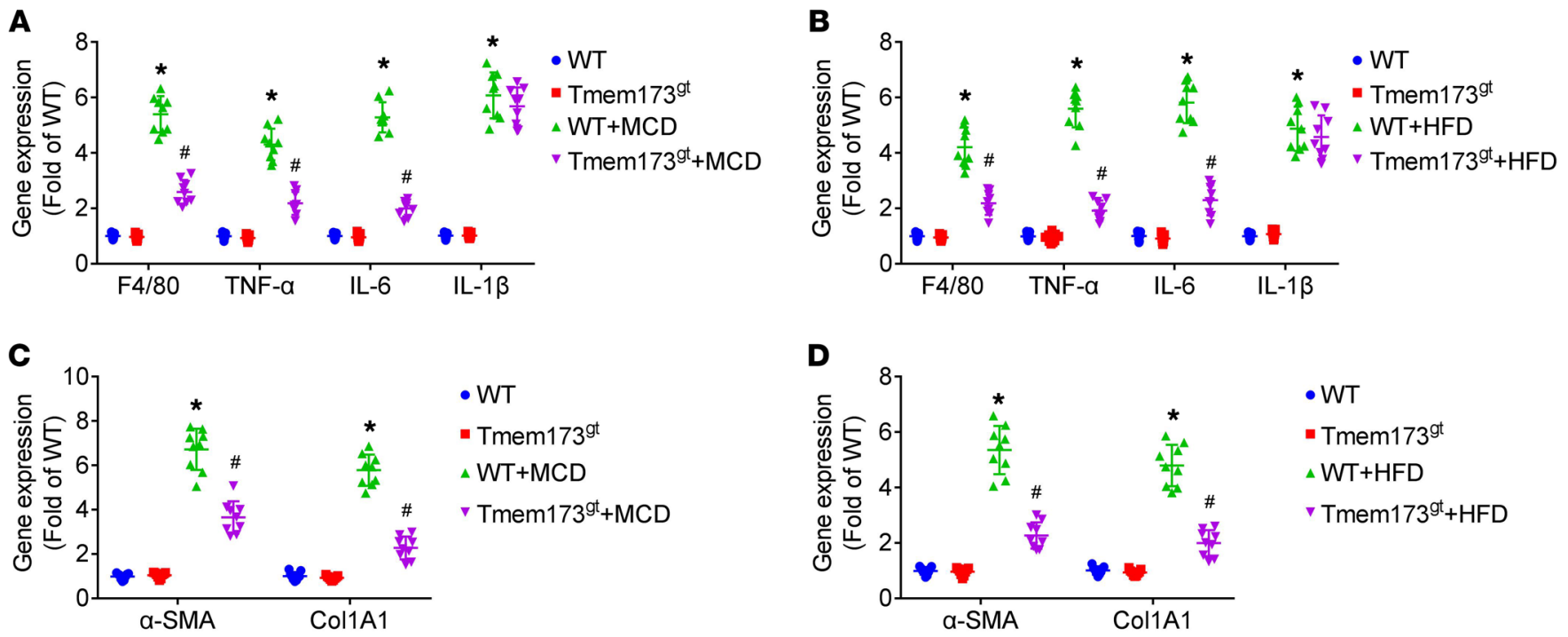

Figure 4. Deficiency of STING attenuated MCD- or HFD-induced inflammation and modulated mRNA expression of genes associated with fibrogenesis in livers of mice. WT or STING-deficient mice (Tmem173"t) were exposed to MCD for 8 weeks or HFD for 26 weeks to induce NASH. Graphs show mRNA expression of F4/80, TNF- $\alpha$, IL-6, and IL-1 $\beta$ ( $\mathbf{A}$ and $\mathbf{B}$ ) and $\alpha-$ SMA and Col1A1 (C and $\mathbf{D}$ ) in livers of mice. $n=9$ in each group. Values are shown as mean \pm SD. ${ }^{*} P<0.05$ versus WT mice fed with control diet; ${ }^{\#} P<0.05$ versus WT mice fed with MCD or HFD. Statistical significance was determined using 1 -way ANOVA followed by Tukey-Kramer multiple comparisons test.

mtDNA from hepatocytes of HFD-fed mice induced activation of $N F-\kappa B$ and increased $m R N A$ expression of TNF- $\alpha$ and $I L-6$. We tested to determine whether an 8-week MCD model or a 26-week HFD model led to an increase in plasma mtDNA. The mtDNA levels, but not nDNA levels, were significantly higher in plasma of MCDfed mice (Supplemental Figure 5A) and HFD (Supplemental Figure $5 \mathrm{~B}$ ) mice than in control mice. Furthermore, Western blotting analysis and immunohistochemistry results revealed that STING protein was not expressed in hepatocytes (Figure 5, A and B). Western blotting results demonstrated that primary murine KCs expressed STING protein.

KCs were isolated from livers of WT or Tmem $173^{\text {gt }}$ mice fed with chow or HFD. The mtDNA (100 ng/ml) (7) from hepatocytes was added to KCs for 12 hours. It was found that exposure to mtDNA from hepatocytes of HFD-fed mice led to increased activity of $\mathrm{NF}-\kappa \mathrm{B}$ (Figure $5 \mathrm{C}$ ) and upregulation of mRNA expression of TNF- $\alpha$ (Figure 5D) and IL-6 (Figure 5E). The mtDNA from hepatocytes of chow-fed mice had no significant effect on the activity of NF- $\kappa \mathrm{B}$ and mRNA expression of TNF- $\alpha$ and IL-6. Increased responsiveness of KCs from HFDfed mice to mtDNA from hepatocytes of HFD-fed mice was observed compared with that from control mice. STING deficiency attenuated NF- $\kappa \mathrm{B}$ activation and upregulation of mRNA expression of TNF- $\alpha$ and IL- 6 induced by mtDNA from hepatocytes of HFD-fed mice. Exposure to mtDNA from hepatocytes of HFD-fed mice led to upregulation of mRNA expression of IL-1 $\beta$ in KCs, but STING deficiency had no significant effect on IL-1 $\beta$ expression (Figure $5 \mathrm{~F}$ ).

The role of STING and TLR9 in the inflammation induced by $m t D N A$. The mtDNA from injured hepatocytes was also recognized by TLR9 as an endogenous ligand $(7,8)$. Transfection with TLR9 shRNA lentiviral particles downregulated TLR9 protein expression in cultured KCs (Supplemental Figure 6). Unlike
STING deficiency, knockdown of TLR9 suppressed IL-1 $\beta$ expression in KCs when exposed to mtDNA from hepatocytes of HFDfed mice. In addition, it was found that STING deficiency and TLR9 knockdown synergistically suppressed expression of TNF- $\alpha$ and IL-6 (Supplemental Figure 7).

The role of IFN regulatory factor 3 and $N F-\kappa B$ in inflammation induced by mtDNA. KCs isolated from livers of WT mice fed with chow or HFD were treated with BAY11-7082 (an NF- $\mathrm{B}$ inhibitor, $10 \mu \mathrm{M}$ ) (18) or Bx-795 (an IFN regulatory factor 3 [IRF3] inhibitor, $1 \mu \mathrm{M}$ ) (19) for 30 minutes. Afterwards, they were stimulated with mtDNA from hepatocytes of HFD-fed mice for 12 hours. It was found that pretreatment with BAY117082 markedly inhibited upregulation of TNF- $\alpha$ (Figure 6, A and B) and IL-6 (Figure 6, C and D) induced by mtDNA (HFD). Pretreatment of KCs with Bx-795 slightly reduced expression of TNF- $\alpha$ and IL- 6 .

$D M X A A$ induced steatosis and inflammation in livers of mice. WT or STING-deficient mice were exposed to 5,6-dimethylxanthenone-4-acetic acid (DMXAA, a known activator of mouse STING, $25 \mathrm{mg} / \mathrm{kg} / 2$ days, i.p.) (20) for 8 weeks. At the end the experiment, DMXAA showed no significant effect on body weight (Figure 7A) or fasting glucose levels (Figure 7B). DMXAA exposure slightly increased serum levels of ALT (Figure 7C) and AST (Figure 7D). DMXAA exposure in mice led to enhanced levels of triglycerides (Figure 7E) and cholesterol (Figure 7F) in livers and upregulated mRNA expression of TNF- $\alpha$ and IL-6 (Figure $7 G$ ), which was abolished by STING deficiency. Similar results were demonstrated in protein levels of TNF- $\alpha$ and IL-6 (Supplemental Figure 8) in livers.

DMXAA induced STING-dependent activation of NF- $\kappa B$ and expression of TNF- $\alpha$ and IL- 6 in cultured KCs. The KCs isolated from WT or STING-deficient mice were stimulated with DMXAA $(20 \mu \mathrm{g} / \mathrm{ml})(21)$ for 6 hours or left unstimulated. DMXAA incu- 
A

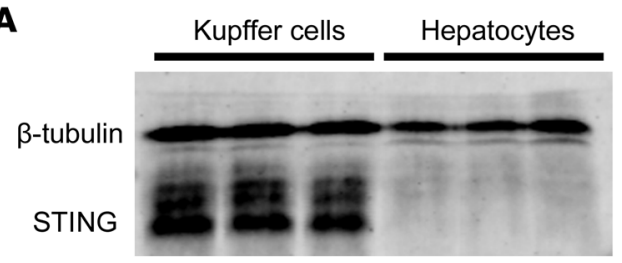

C

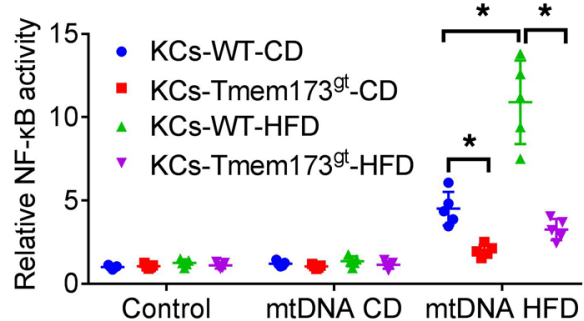

$\mathbf{E}$

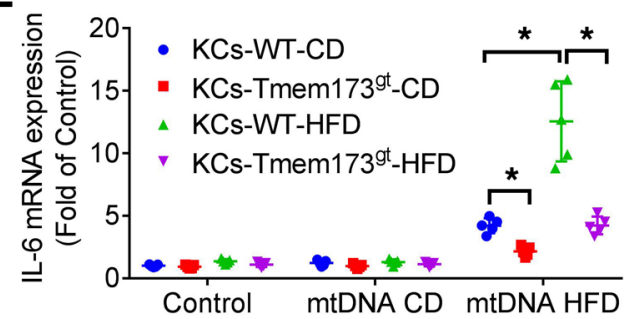

B

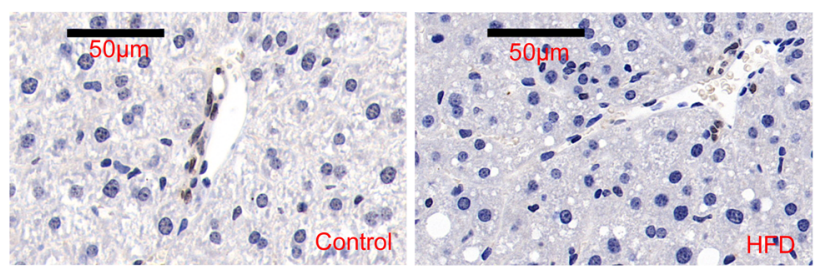

D

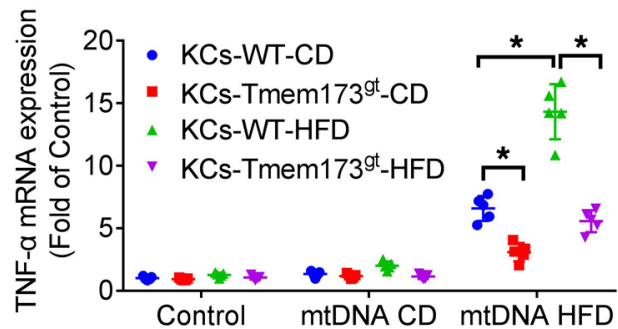

$\mathbf{F}$

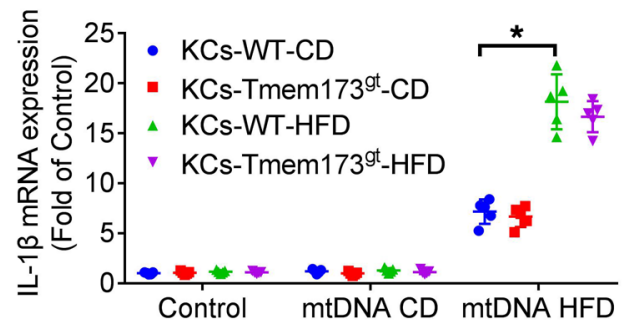

Figure 5. mtDNA from hepatocytes of HFD-fed mice induced activation of NF- $\kappa B$ and increased mRNA expression of TNF- $\alpha$ and IL-6. Primary hepatocytes and KCs were isolated from C57BL/6 mice. Protein expression of STING (A) was detected in KCs, but not in hepatocytes. The $\beta$-tubulin was used as a loading control. Liver sections of mice fed with control chow or HFD were stained for STING (B). The mtDNA (100 ng/ml) from hepatocytes of chow-fed (mtDNA CD) and HFD-fed (mt DNA HFD) mice was added to KCs from WT or STING-deficient mice (Tmem173"st) fed with chow or HFD for 12 hours. The activity of NF- $\kappa B$ (C) was determined by luciferase assay, and mRNA expression of TNF- $\alpha(\mathbf{D})$, IL- 6 (E), and IL-1 $\beta$ (F) was determined by RT-PCR. The in vitro experiments were performed 5 times, and each experiment was performed with replicates. ${ }^{*} P<0.05$. Statistical significance was determined using 1 -way ANOVA followed by Tukey-Kramer multiple comparisons test.

bation enhanced activity of NF-kB (Figure $8 \mathrm{~A}$ ) and upregulated expression of TNF- $\alpha$ (Figure 8, B and D) and IL-6 (Figure 8, C and E) in cultured KCs; this was abolished by STING deficiency.

\section{Discussion}

We used STING-deficient (Tmem173"t) mice, in which a point mutation in the Sting gene (T596A) led to STING dysfunction and degradation (22), and we found that STING deficiency markedly attenuated hepatic steatosis, fibrosis, and inflammation in both MCD- and HFD-fed murine models in our work.

One recent report claimed that the early proapoptotic activation of IRF3 by $\mathrm{CCl}_{4}$ was hepatocyte specific and mediated by STING (13). The authors also claimed that STING and IRF3 were key determinants of alcoholic liver disease, linking ER stress signaling with hepatocyte apoptosis (14). Qiao et al. reported that knockdown of STING in LO2 cells (a normal human fetal liver cell line) attenuated free fatty acid-induced apoptosis (23). Cho et al. reported that lipotoxicity induced hepatic protein inclusions through STING/TBK1 activation in HepG2 cells (a human hepatocellular carcinoma cell line) (24). However, while IRF3 is mainly expressed in hepatocytes (13), STING protein was not expressed in hepatocytes of adult humans or mice $(25,26)$. In our work, Western blotting analysis and immunohistochemistry results also demonstrated that hepatocytes in livers of adult mice did not express STING protein.
In the investigation of the role of STING in NASH, mononuclear cells (particular for KCs) are of critical importance. KCs are a crucial part of the innate immune system, acting as scavengers and phagocytes in livers (27). KCs were the first cells responding to hepatocyte injuries, leading to activation of NF- $\mathrm{kB}$ and induction of proinflammatory cytokines (TNF- $\alpha$, IL-1 $\beta$, and IL-6) and chemokine and monocyte recruitment (28), and early depletion of liver KCs prevented the development of NASH in animals (29, 30). In our work, STING deficiency attenuated mtDNA-induced activation of NF- $\mathrm{\kappa B}$ and induction of TNF- $\alpha$ and IL-6 in KCs. The increase of TNF- $\alpha$-producing KCs was crucial for NASH development, acting by promoting blood monocyte infiltration through the production of IP-10 and MCP-1 (28). Intraperitoneal injection of TNF- $\alpha$ dramatically accelerated the hepatic accumulation of fat by upregulating gene expression of SREBP-1c (31). Macrophage-derived TNF- $\alpha$ enhanced the survival of activated hepatic stellate cells (HSCs) and thereby increased liver fibrosis (32). In addition, recombinant or macrophage-derived IL- 6 was a potent activator of the lipogenic factors in hepatocytes $(33,34)$. Therefore, STING might participate in hepatic steatosis, fibrosis, and inflammation through promoting activation of NF- $\mathrm{\kappa B}$ and induction of TNF- $\alpha$ and IL- 6 in KCs.

It is known that STING utilizes IRF3 and NF-kB pathways to exert its effects on type I IFN and proinflammatory genes (35). However, it was reported that global KO of IRF3 significantly pro- 
A - Vehicle

- BAY 11-7082

$\triangle$ BX-795

- BAY 11-7082 plus Bx-795

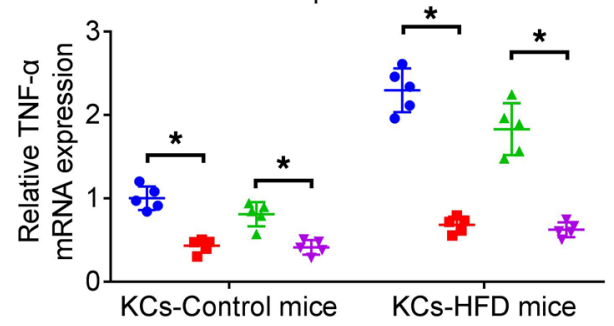

C Vehicle

- BAY 11-7082

$\triangle$ Bx-795

- BAY 11-7082 plus Bx-795

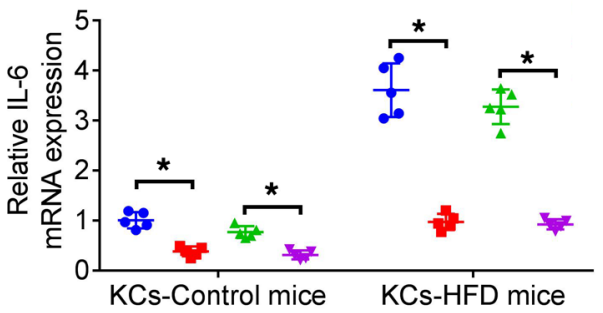

B

- Vehicle

- BAY 11-7082

- Bx-795

v BAY 11-7082 plus Bx-795

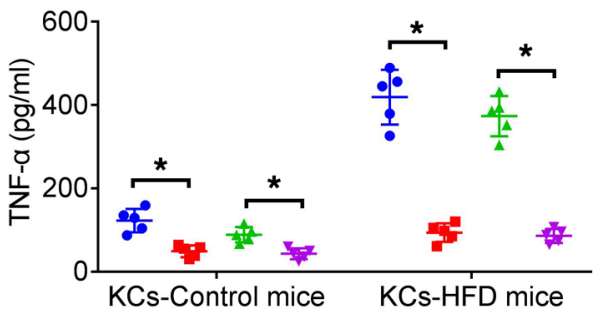

D

- Vehicle

- BAY 11-7082

$\triangle$ Bx-795

- BAY 11-7082 plus Bx-795

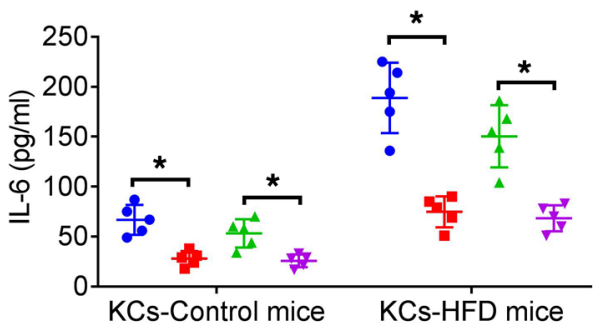

Figure 6. The role of NF-KB and IRF3 in inflammation induced by mtDNA in KCs. mtDNA (100 ng/ml) from hepatocytes of HFD-fed (mtDNA HFD) mice was added to KCs from WT or STING-deficient mice (Tmem173 $3^{\text {gt }}$ ) fed with chow or HFD for 12 hours in the presence of pretreatment with the NF- $K B$ inhibitor (BAY11-7082, $10 \mu \mathrm{M})$ or IRF3 inhibitor (Bx-795, $1 \mu \mathrm{M})$ for 30 minutes. Cell lysates and culture supernatant were collected to measure TNF- $\alpha$ and IL-6 mRNA levels by real-time PCR (A and $\mathbf{C}$ ) or protein levels by ELISA (B and $\mathbf{D})$. The in vitro experiments were performed 5 times, and each experiment was performed with replicates. ${ }^{*} P<0.05$. Statistical significance was determined using 1 -way ANOVA followed by Tukey-Kramer multiple comparisons test.

moted chronic HFD-induced hepatic steatosis (36), indicating that STING-mediated inflammation in NASH might not be dependent on IRF3. STING-mediated IRF3 and NF- $\mathrm{BB}$ activation could be uncoupled from each other in bone marrow-derived macrophages and bone marrow-derived dendritic cells (19). In addition, reduced expression of IRF3 (36) and activated NF- $\kappa B$ (37) were observed in animals or patients with NASH. Therefore, in fatty livers, STING/NF- $\mathrm{KB}$ might be the principal pathway contributing to KC-derived inflammation.

The mtDNA from injured hepatocytes was also recognized by TLR9 as an endogenous ligand, and subsequent IL-1 $\beta$ production was important for the development of NASH $(7,8)$. Both STING and TLR9 were located in the endoplasmic reticulum, and our work revealed that STING deficiency had no significant effect on IL-1 $\beta$ production in NASH mice and cultured KCs. STING deficiency and TLR9 knockdown synergistically suppressed expression of TNF- $\alpha$ and IL- 6 in KCs when exposed to mtDNA. Thus, STING and TLR9 in KCs might synergistically contribute to mtDNA-induced inflammation in NASH.

In the present work, deficiency of STING attenuated HFDinduced moderate insulin resistance and weight gain in mice. Interestingly, in contrast with a previous report (15), STINGdeficient mice in our work showed higher fasting glucose levels and lower body weight, accompanied by unaltered insulin levels. It was reported that IRF3 KO mice also showed higher fasting glycemia
(36) and glycemia measured in the fed state (38), accompanied by unaltered insulin levels. However, IRF3 KO mice showed higher body weight (36), which was not consistent with STING-deficient mice. STING might modulate glucose levels through the IRF3 pathway, but modulate body weight independently of IRF3. Further investigation is required to elucidate the underlying mechanism.

Finally, one limitation should be noted in our work. STING was also expressed in endothelial cells. STING knockdown suppressed palmitic acid-induced endothelial inflammation and attenuated monocyte/endothelial cell adhesion (15). It was reported that liver sinusoidal endothelial cell injury might have a "gatekeeper" role in the progression from simple steatosis to the early NASH stage (39). STING in liver sinusoidal endothelial cells might be important as well in the development of NASH, which requires further investigation.

In conclusion, we proposed that STING functions as an mtDNA sensor in the KCs of liver under lipid overload and induces NF- $\kappa$ Bdependent inflammation in NASH. Development of STING inhibitors or manipulation of the expression of STING may represent a novel approach to managing NASH in patients.

\section{Methods}

Animals. WT mice and STING-deficient (Tmem173 ${ }^{\text {gt }}$ ) mice were purchased from Jackson Laboratory. All mice used were on a C57BL/6J background. 
A

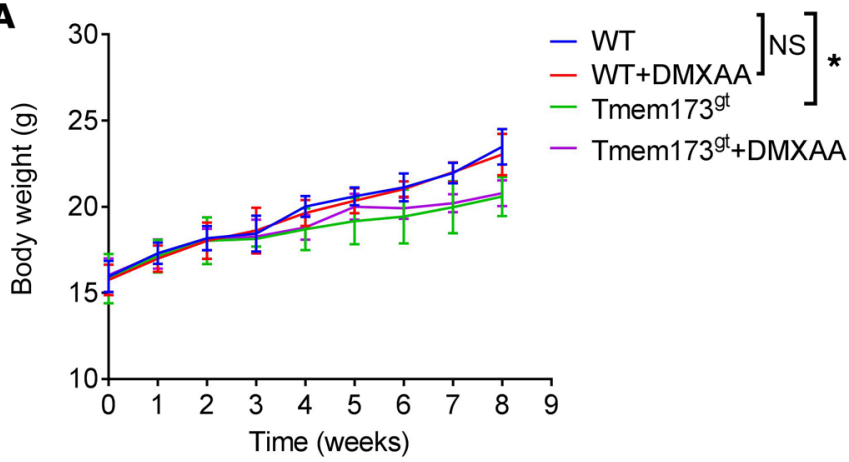

B

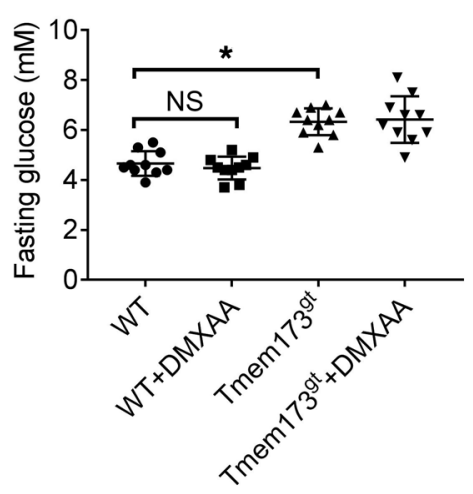

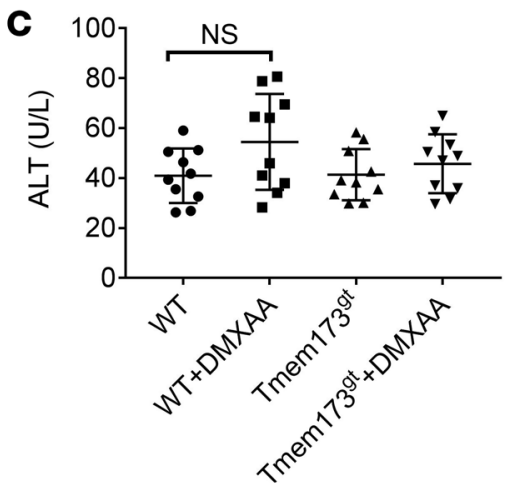
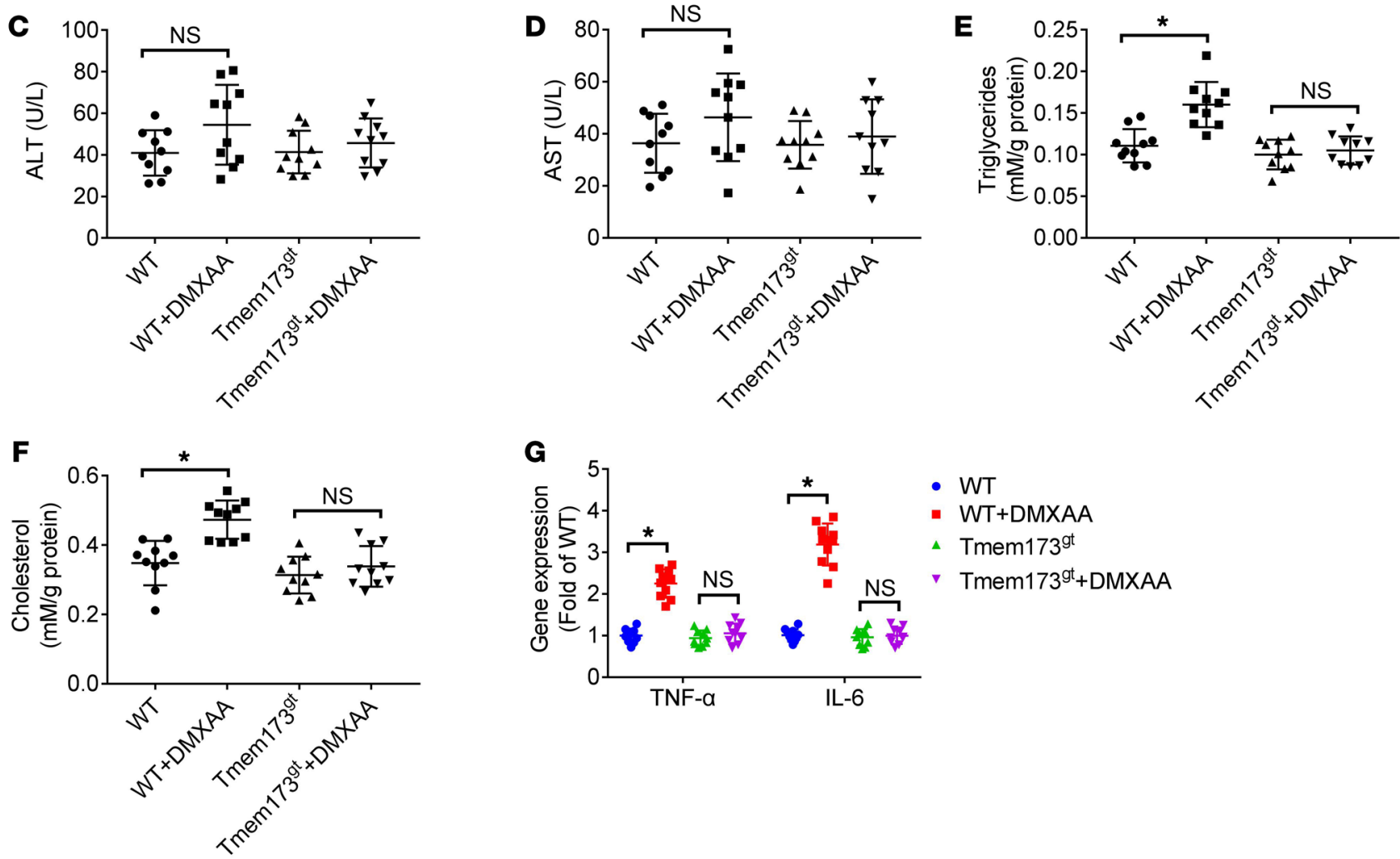

Figure 7. DMXAA induced steatosis and inflammation in livers of mice. WT or STING-deficient mice (Tmem173 ${ }^{\text {gt }}$ ) were exposed to DMXAA (a known activator of mouse STING, $25 \mathrm{mg} / \mathrm{kg} / 2$ days, i.p.) for 8 weeks. Graphs show the body weight (A), levels of fasting glucose (B), levels of ALT (C) and AST (D) in serum, and levels of triglycerides (E) and cholesterol (F) and mRNA expression of TNF- $\alpha$ and IL-6 (G) in livers. $n=10$ in each group. Values are shown as mean \pm SD. ${ }^{*} P<0.05$. Statistical significance was determined using 1-way ANOVA followed by Tukey-Kramer multiple comparisons test.

Adult female mice (6 weeks old) were bred in-house in a pathogen-free facility and fed either a control diet $(59.4 \%$ carbohydrate, $4.3 \%$ lipid, and 24.3 protein) or HFD ( $2 \%$ cholesterol, $0.2 \%$ cholic acid, $8 \%$ yolk powder, $18 \%$ lard; $43.0 \%$ carbohydrate, $25.9 \%$ lipid, and $19.7 \%$ protein) ad libitum for 26 weeks.

Adult female mice (6 weeks old) were bred in-house in a pathogen-free facility and fed either control diet or MCD (composition of diet is shown in Supplemental Methods, Supplemental Table 1; TROPHIC Animal Feed High-tech Co.) ad libitum for 8 weeks. At the end of the experiment, the mice were anesthetized with $3 \%$ sodium pentobarbital, and their livers were immediately harvested.

Details of real-time quantitative PCR (RT-PCR), Western blotting, biochemical analysis, and histological analysis are shown in Sup- plemental Methods. Unless otherwise specified, drugs and reagents were purchased from Sigma-Aldrich.

Cell isolation and treatment. Hepatocytes from WT mice fed with chow or HFD were isolated by collagenase perfusion as described previously (40). Viability was more than $90 \%$ for all preparations, as determined by trypan blue staining. Fresh hepatocytes were seeded on collagen-coated 6-well plates. Cells were washed after 3 hours to remove dead cells, and fresh culture medium was added to incubate the cells overnight. Mitochondrial fractions were extracted from hepatocytes with the Mitochondria Isolation Kit for Cultured Cells (Thermo Fisher Scientific).

Mouse KCs were isolated from WT or Tmem173 ${ }^{\text {gt }}$ mice fed with chow or HFD by the density gradient separation of Percoll (GE Healthcare Life Sciences), and then plates were gently washed 
A
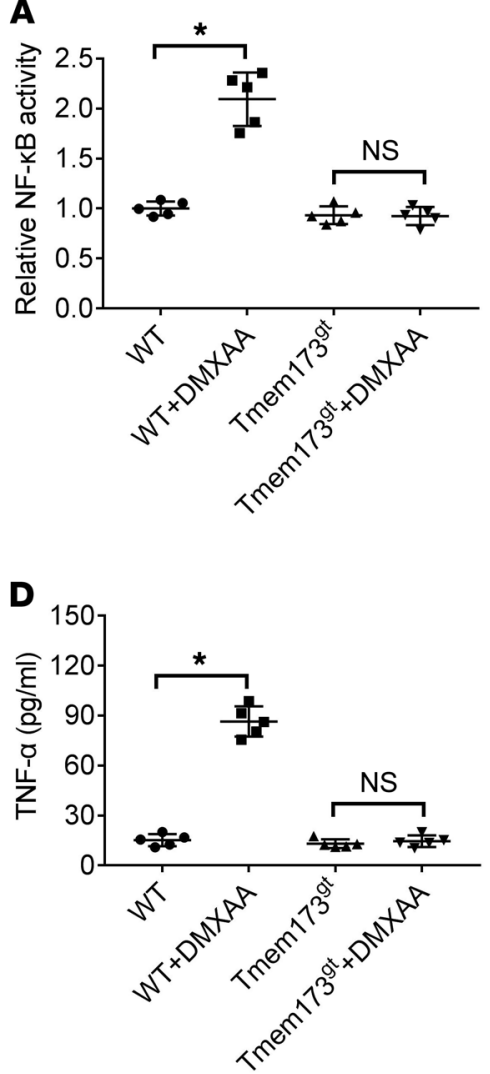

B

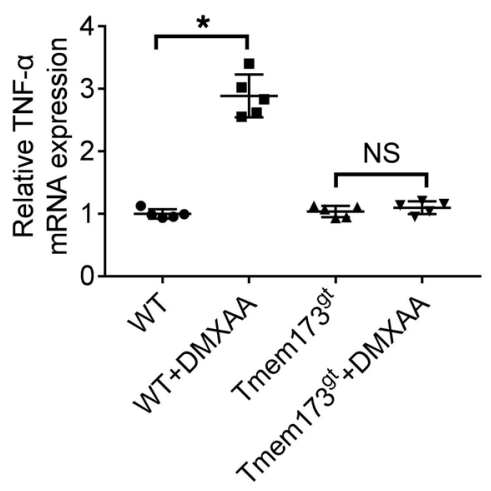

$\mathbf{E}$

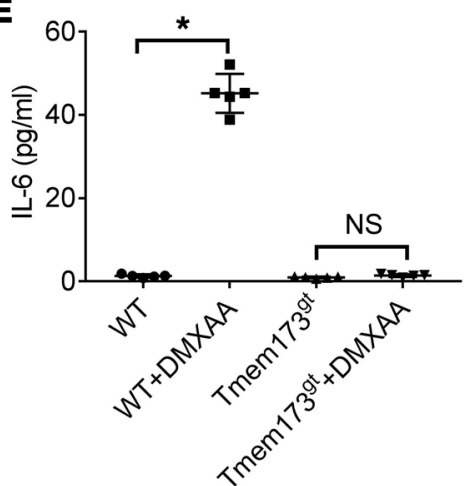

C

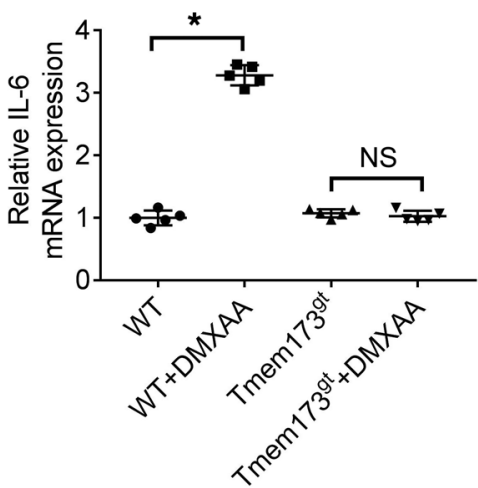

Figure 8. DMXAA induced activation of NF- $\mathrm{B}$ and expression of TNF- $\alpha$ and IL-6 in cultured KCs. The KCs isolated from WT or STING-deficient mice (Tmem173 ${ }^{\text {gt }}$ ) were stimulated with DMXAA $(20 \mu \mathrm{g} / \mathrm{ml})$ for 6 hours or not. The activity of NF- $\mathrm{KB}(\mathbf{A})$ was determined by luciferase assay. The cell lysates and culture supernatant were collected 6 hours after DMXAA treatment to look at TNF- $\alpha$ and IL-6 mRNA levels by real-time PCR (B and C) or protein levels by ELISA ( $\mathbf{D}$ and $\mathbf{E}$ ). Values are shown as mean \pm SD. The in vitro experiments were performed 5 times, and each experiment was performed with replicates. ${ }^{*} P<0.05$. Statistical significance was determined using 1-way ANOVA followed by Tukey-Kramer multiple comparisons test.

and media was replenished after seeding cells for 2 hours to raise KC purity. Then KCs were isolated by using (a) APC-conjugated rat anti-mouse CD11b (BioLegend, catalog 101212; 1:100) and anti-APC magnetic beads (Miltenyi Biotec, catalog 130-090-855) and (b) the depleted CD11b fraction was stained with APC-conjugated rat antimouse F4/80 antibody (BioLegend, catalog 123116; 1:100) and antiAPC magnetic beads (Miltenyi Biotec, catalog 130-090-855). Cell viability ( $>90 \%)$ was evaluated by trypan blue exclusion.

Statistics. The values are shown as mean $\pm \mathrm{SD}$. One-way ANOVA followed by Tukey-Kramer multiple comparisons test was performed for the statistical analysis. $P<0.05$ was considered to be statistically significant.

Study approval. All mice used received humane care in compliance with institutional animal care guidelines, and protocols were approved by the local institutional committee. All protocols were conducted in accordance with the Guide for the Care and Use of Laboratory Animals (National Academies Press, 2011). All efforts were made to minimize the number of mice used and their suffering.

\section{Author contributions}

YY, YZ, YL, and XZ conceived and designed the experiments. YY, YZ, YL, WA, and JS collected, analyzed, and interpreted data. YY and $\mathrm{XZ}$ drafted the article.

\section{Acknowledgments}

This work was supported by grants from the National Natural Science Foundation of China (81600193 to YY; 81570208 to XZ; 81700396 to YL).

Address correspondence to: Yuefan Zhang, Department of Clinical Pharmacy, Shanghai General Hospital, Shanghai Jiaotong University, Haining Road 100, Shanghai 200025, China. Or to: Xianxian Zhao, Department of Cardiovasology, Changhai Hospital, Second Military Medical University, Changhai Road 168, Shanghai, 200433, China. Phone: 862131161245; Email: zhangyuefan@126.com (YZ); xianxianz2010@163.com (XZ).
1. Fan JG, Kim SU, Wong VW. New trends on obesity and NAFLD in Asia. J Hepatol. 2017;67(4):862-873.

2. Arrese M, Cabrera D, Kalergis AM, Feldstein AE. Innate immunity and inflammation in NAFLD/ NASH. Dig Dis Sci. 2016;61(5):1294-1303.

3. Wenfeng Z, Yakun W, Di M, Jianping G, Chuanx- in W, Chun H. Kupffer cells: increasingly significant role in nonalcoholic fatty liver disease. Ann Hepatol. 2014;13(5):489-495.

4. Garcia-Martinez I, Shaker ME, Mehal WZ. Therapeutic opportunities in damage-associated molecular pattern-driven metabolic diseases. Antioxid Redox Signal. 2015;23(17):1305-1315.

5. West AP, Shadel GS. Mitochondrial DNA in innate immune responses and inflammatory pathology. Nat Rev Immunol. 2017;17(6):363-375. 6. Koliaki C, et al. Adaptation of hepatic mitochon- 
drial function in humans with non-alcoholic fatty liver is lost in steatohepatitis. Cell Metab. 2015;21(5):739-746.

7. Garcia-Martinez I, et al. Hepatocyte mitochondrial DNA drives nonalcoholic steatohepatitis by activation of TLR9. JClin Invest. 2016;126(3):859-864.

8. Miura K, et al. Toll-like receptor 9 promotes steatohepatitis by induction of interleukin-1beta in mice. Gastroenterology. 2010;139(1):323-34.e7.

9. Rongvaux A, et al. Apoptotic caspases prevent the induction of type I interferons by mitochondrial DNA. Cell. 2014;159(7):1563-1577.

10. White MJ, et al. Apoptotic caspases suppress mtDNA-induced STING-mediated type I IFN production. Cell. 2014;159(7):1549-1562.

11. Lood C, et al. Neutrophil extracellular traps enriched in oxidized mitochondrial DNA are interferogenic and contribute to lupus-like disease. Nat Med. 2016;22(2):146-153.

12. Zhang X, et al. Cyclic GMP-AMP containing mixed phosphodiester linkages is an endogenous high-affinity ligand for STING. Mol Cell. 2013;51(2):226-235.

13. Iracheta-Vellve A, et al. Endoplasmic reticulum stress-induced hepatocellular death pathways mediate liver injury and fibrosis via stimulator of interferon genes. J Biol Chem. 2016;291(52):26794-26805.

14. Petrasek J, et al. STING-IRF3 pathway links endoplasmic reticulum stress with hepatocyte apoptosis in early alcoholic liver disease. Proc Natl Acad Sci USA. 2013;110(41):16544-16549.

15. Mao Y, et al. STING-IRF3 triggers endothelial inflammation in response to free fatty acid-induced mitochondrial damage in diet-induced obesity. Arterioscler Thromb Vasc Biol. 2017;37(5):920-929.

16. Rinella ME, Green RM. The methionine-choline deficient dietary model of steatohepatitis does not exhibit insulin resistance. J Hepatol. 2004;40(1):47-51.

17. Larter CZ, Yeh MM. Animal models of NASH: getting both pathology and metabolic context right. J Gastroenterol Hepatol. 2008;23(11):1635-1648.

18. Park SY, et al. RhoA/ROCK-dependent pathway is required for TLR2-mediated IL-23 production in human synovial macrophages: suppression by cilostazol. Biochem Pharmacol. 2013;86(9):1320-1327.

19. Blaauboer SM, Gabrielle VD, Jin L. MPYS/ STING-mediated TNF- $\alpha$, not type I IFN, is essential for the mucosal adjuvant activity of (3'-5')-cyclic-di-guanosine-monophosphate in vivo. J Immunol. 2014;192(1):492-502.

20. Guo F, et al. STING agonists induce an innate antiviral immune response against hepatitis B virus. Antimicrob Agents Chemother. 2015;59(2):1273-1281.

21. Guo X, et al. Cyclic GMP-AMP ameliorates diet-induced metabolic dysregulation and regulates proinflammatory responses distinctly from STING activation. Sci Rep. 2017;7(1):6355.

22. Sauer JD, et al. The N-ethyl-N-nitrosoureainduced Goldenticket mouse mutant reveals an essential function of Sting in the in vivo interferon response to Listeria monocytogenes and cyclic dinucleotides. Infect Immun 2011;79(2):688-694.

23. Qiao JT, et al. Activation of the STING-IRF3 pathway promotes hepatocyte inflammation, apoptosis and induces metabolic disorders in nonalcoholic fatty liver disease. Metab Clin Exp. 2018;81:13-24.

24. Cho CS, et al. Lipotoxicity induces hepatic protein inclusions through TANK binding kinase 1-mediated p62/sequestosome 1 phosphorylation. Hepatology. 2018;68(4):1331-1346.

25. Thomsen MK, et al. Lack of immunological DNA sensing in hepatocytes facilitates hepatitis B virus infection. Hepatology. 2016;64(3):746-759.

26. Lei Z, et al. cGAS-mediated autophagy protects the liver from ischemia-reperfusion injury independently of STING. Am J Physiol Gastrointest Liver Physiol. 2018;314(6):G655-G667.

27. Malhi H, Gores GJ. Molecular mechanisms of lipotoxicity in nonalcoholic fatty liver disease. Semin Liver Dis. 2008;28(4):360-369.

28. Tosello-Trampont AC, Landes SG, Nguyen V, Novobrantseva TI, Hahn YS. Kuppfer cells trigger nonalcoholic steatohepatitis development in diet-induced mouse model through tumor necrosis factor- $\alpha$ production. J Biol Chem. 2012;287(48):40161-40172.

29. Zeng TS, Liu FM, Zhou J, Pan SX, Xia WF, Chen LL. Depletion of Kupffer cells attenuates system- ic insulin resistance, inflammation and improves liver autophagy in high-fat diet fed mice. Endocr J. 2015;62(7):615-626.

30. Yang YY, et al. Kupffer cell depletion attenuates leptin-mediated methoxamine-stimulated portal perfusion pressure and thromboxane $\mathrm{A} 2$ release in a rodent model of NASH-cirrhosis. Clin Sci. 2012;123(12):669-680.

31. Endo M, Masaki T, Seike M, Yoshimatsu H. TNF-alpha induces hepatic steatosis in mice by enhancing gene expression of sterol regulatory element binding protein-1c (SREBP-1c). Exp Biol Med (Maywood). 2007;232(5):614-621.

32. Pradere JP, et al. Hepatic macrophages but not dendritic cells contribute to liver fibrosis by promoting the survival of activated hepatic stellate cells in mice. Hepatology. 2013;58(4):1461-1473.

33. Dembek A, et al. Hepatic interleukin-6 production is maintained during endotoxin tolerance and facilitates lipid accumulation. Immunobiology. 2017;222(6):786-796.

34. Vida M, et al. Chronic administration of recombinant IL-6 upregulates lipogenic enzyme expression and aggravates high-fat-diet-induced steatosis in IL-6-deficient mice. Dis Model Mech. 2015;8(7):721-731.

35. Paludan SR, Bowie AG. Immune sensing of DNA. Immunity. 2013;38(5):870-880.

36. Wang XA, et al. Interferon regulatory factor 3 constrains IKK $\beta / \mathrm{NF}-\kappa \mathrm{B}$ signaling to alleviate hepatic steatosis and insulin resistance. Hepatology. 2014;59(3):870-885.

37. Willy JA, Young SK, Stevens JL, Masuoka HC, Wek RC. CHOP links endoplasmic reticulum stress to NF- $\mathrm{kB}$ activation in the pathogenesis of nonalcoholic steatohepatitis. Mol Biol Cell. 2015;26(12):2190-2204.

38. Dogusan Z, et al. Double-stranded RNA induces pancreatic beta-cell apoptosis by activation of the toll-like receptor 3 and interferon regulatory factor 3 pathways. Diabetes. 2008;57(5):1236-1245.

39. Miyao M, et al. Pivotal role of liver sinusoidal endothelial cells in NAFLD/NASH progression. Lab Invest. 2015;95(10):1130-1144.

40. Yang G, Lee HE, Lee JY. A pharmacological inhibitor of NLRP3 inflammasome prevents non-alcoholic fatty liver disease in a mouse model induced by high fat diet. Sci Rep. 2016;6:24399. 